\title{
Tropospheric delay determination by Kalman filtering VLBI data
}

\author{
Benedikt Soja $\mathbb{1 0}^{*}$, Tobias Nilsson, Maria Karbon, Florian Zus, Galina Dick, Zhiguo Deng, Jens Wickert, \\ Robert Heinkelmann and Harald Schuh
}

\begin{abstract}
The troposphere is one of the most important error sources for space geodetic techniques relying on radio signals. Since it is not possible to model the wet part of the tropospheric delay with sufficient accuracy, it needs to be estimated from the observational data. In the analysis of very long baseline interferometry (VLBI) data, the parameter estimation is routinely performed using a least squares adjustment. In this paper, we investigate the application of a Kalman filter for parameter estimation, specifically focusing on the tropospheric delays. The main advantages of a Kalman filter are its real-time capability and stochastic approach. We focused on the latter and derived stochastic models for VLBI zenith wet delays, taking into account temporal and location-based differences. Compared to a static noise model, the quality of station coordinates, also estimated in the Kalman filter, increased as a result. In terms of baseline length and station coordinate repeatabilities, this improvement amounted to $2.3 \%$. Additionally, we compared the Kalman filter and least squares results for VLBI with zenith wet delays derived from GPS (Global Positioning System), water vapor radiometers, and ray tracing in numerical weather models. The agreement of the Kalman filter VLBI solution with respect to water vapor radiometer data was larger than that of the least squares solution by 6-15\%. Our investigations are based on selected VLBI data (CONT campaigns) that are closest to how future VLBI infrastructure is designed to operate. With the aim for continuous and near real-time parameter estimation and the promising results which we have achieved in this study, we expect Kalman filtering to grow in importance in VLBI analysis.
\end{abstract}

Keywords: Geodesy; VLBI; Troposphere; Zenith wet delays; Kalman filter; Stochastic modelling

\section{Background}

Very long baseline interferometry (VLBI, Schuh and Behrend 2012; Schuh and Böhm 2013), among other space geodetic techniques, has been successfully used in the past to estimate tropospheric parameters, in particular zenith wet delays (ZWD) and horizontal delay gradients (e.g., Heinkelmann et al. 2011). The standard method for parameter estimation in operational VLBI analysis is the least squares adjustment, also called least squares method (LSM). Another option is the use of a Kalman filter (KF, Kalman 1960). Pioneering work about the application of a Kalman filter in VLBI analysis was performed by Herring et al. (1990), with the focus on tropospheric investigations by Tralli et al. (1992). However, while the theoretical examinations were deep and manifold, the observational

*Correspondence: bsoja@gfz-potsdam.de

GFZ German Research Centre for Geosciences, Telegrafenberg, D-14473 Potsdam, Germany data used in those studies were limited to only a couple of stations and days. Since then, research on using a KF in VLBI analysis has been practically non-existent, except for studies by Pany et al. (2007, 2011), which focused solely on simulations of VLBI observations. This lack is surprising as Kalman filtering has been successfully used in geodetic Global Navigation Satellite Systems (GNSS) analysis (Schüler 2001; Webb and Zumberge 1993), gravity field studies (Kurtenbach et al. 2009), and for combination of space geodetic techniques to derive Earth Orientation Parameters (EOP, Gross 2000; Gross et al. 1998) or terrestrial reference frames (TRF, Wu et al. 2014). Examples of Kalman filtering in GPS data processing for tropospheric investigations are studies by Jarlemark et al. (1998), Emardson and Jarlemark (1999), as well as Schüler (2001).

The advantages of a KF with respect to a least squares adjustment are, among others, its real-time capability and stochastic modeling of parameters. The former will

\section{Springer}

(c) 2015 Soja et al. Open Access This article is distributed under the terms of the Creative Commons Attribution 4.0 International License (http://creativecommons.org/licenses/by/4.0/), which permits unrestricted use, distribution, and reproduction in any medium, provided you give appropriate credit to the original author(s) and the source, provide a link to the Creative Commons license, and indicate if changes were made. 
be very important in view of the new generation of VLBI infrastructure that is currently in preparation, the VLBI Global Observing System (VGOS, Hase et al. 2012). Today, most data are correlated and made available for analysis several days or weeks after the observation. With VGOS, it is planned that the observational data from the individual radio telescopes is streamed to the correlator continuously and in near real time (Petrachenko et al. 2009). Accordingly, future VLBI analysis software packages will need to be able to produce results in real time as well, for instance, by using Kalman filtering.

However, also for post processing, where the current focus of VLBI analysis lies, the application of a KF is justified. When considering physical effects that show a stochastic behavior, the deterministic approach of LSM is not optimal. In particular for troposphere and clock parameters, the stochastic approach of a KF is closer to reality. Any improvement in the handling of tropospheric effects is welcome as they are the most significant source of errors in VLBI analysis (Pany et al. 2011) and should thus help to improve other estimated parameters like station coordinates, which are of particularly great interest in geodesy, as well. However, also the tropospheric delays from VLBI are sought to be derived with highest possible quality as they are useful for climatic studies (Heinkelmann et al. 2007) or could in the future possibly help to improve numerical weather models.

For these reasons, we have used a KF to estimate zenith wet delays (ZWD) from VLBI data. Other than the studies by Herring et al. (1990) and Tralli et al. (1992), our focus lies on the comparison of KF and LSM solutions, based on a much extended and up-to-date set of observational data, and the effect of different ZWD noise models on station coordinates. In the "Methods" section, the Kalman filter setup and noise characterization, as well as the LSM solution, are described. The "Data" section gives information on the used VLBI and external data sets. In the following section, the results comprising sophisticated stochastic ZWD models, the effect on station coordinates, and comparisons to external data sets are presented and analyzed.

\section{Methods}

We use the Kalman filter which has been implemented into the VLBI analysis software VieVS@GFZ, a fork from the Vienna VLBI Software (VieVS, Böhm et al. 2012). The KF follows the latest IERS (International Earth Rotation and Reference Systems Service) Conventions (2010) and allows the estimation of station and radio source coordinates, the complete set of EOP, clock offsets, as well as tropospheric ZWD and gradients. For post processing, the filter is run forwards and backwards, followed by a smoothing operation. All parameters are modeled as random walk processes by default.
As the clock offsets are orders of magnitude larger than the other parameters, they are estimated in a first solution and subtracted before the main solution is processed for numerical reasons. The first solution can be obtained by fitting a quadratic polynome in a least squares adjustment or by modeling the clock as an integrated random walk with small process noise in a Kalman filter. Both approaches support the handling of clock breaks and lead to comparable results, but for consistency with the LSM solution, we used the first one. In the main KF solution, the clock offset variations on shorter time scales are estimated by assuming random walk processes.

For tropospheric gradients, both random walk and firstorder Gauss-Markov processes have been tested, with insigificant differences in ZWD and other parameters. Here, the solutions with random walk gradients are presented.

Stations with coordinates in the ITRF2008 (Altamimi et al. 2011) and radio sources that are defining sources of the ICRF2 (Fey et al. 2009) are included in the datum definition by applying appropriate no-net-translation and no-net-rotation conditions. The datum constraints are introduced as additional observations at every epoch with uncertainties of $1 \mathrm{~cm}$ for station and 1 mas for source coordinates, respectively. As a result, the datum conditions are fulfilled at the level of $0.01 \mathrm{~mm}$ for translations and $1 \mu$ as for rotations at every epoch. More details about the Kalman filter implementation can be found in Nilsson et al. (2015).

\section{Stochastic model of the Kalman filter}

The decisive part of the Kalman filter setup is the stochastic model. The choice of the magnitude of the white noise which drives the stochastic processes can have a significant impact on the quality of the results. The state transition equation of the Kalman filter can be written as

$$
x(t+\Delta t)=F(t) x(t)+w(t)
$$

with the state vector $x$, the state transition matrix $F$, the error of prediction $w$ (a white noise process), the epoch $t$, and the time difference $\Delta t$ between the predicted and the current epoch (Gelb 1974). The covariance matrix of the prediction error $Q$ is defined as

$$
Q(t) \equiv\left\langle w(t) w(t)^{T}\right\rangle
$$

with $\langle\cdot\rangle$ denoting the expectation value. For a random walk process and short observation intervals, $Q(t) \approx \Phi \Delta t$ holds with $\Phi$ being the power spectral density (PSD) or the so-called variance rate of the underlying white noise process $w$ (Herring et al. 1990). Especially for the troposphere, efforts have been taken to use carefully tuned noise models, i.e., by applying different values of $\Phi$ for different VLBI stations and observation times. 
Several ways exist to determine the type of a specific stochastic process and consequently the PSD of its driving white noise process (Herring et al. 1990; Schüler 2001). Here, we use the two-sample Allan standard deviation (ASD) $\sigma_{y}$ (Allan 1966) for noise characterization. Following Herring et al. (1990), it is defined by

$$
\sigma_{y}^{2}(\tau)=\left\langle x^{2}(t+\tau)-2 x(t+\tau) x(t)+x^{2}(t)\right\rangle / \tau^{2}
$$

with $\tau$ denoting the time shift. Most stochastic processes can be approximated by $\sigma_{y} \propto \tau^{k}$, at least over a certain range of $\tau$. When plotting $\log \left(\sigma_{y}\right)$ against $\log (\tau)$, a white noise process has a slope $k$ of -1 , a random walk process $-1 / 2$, the turbulence model by Treuhaft and Lanyi (1987) about $-2 / 3$ for $\tau>100$ s. (For comparison, the structure function $D$, employed in other studies, e.g., Herring et al. (1990); Jarlemark and Elgered (1998), shows slopes of $0,1,2 / 3$ for the respective processes.) The PSD, a similar device in the frequency domain, is constant for white noise processes. For random walks, it is in theory not defined but shows a dependency on the frequency to the power of -2 when estimated.

If a random walk is assumed, the variance rate $\Phi$ needed for $Q$ can be estimated by $\Phi=\sigma_{y}^{2}(\tau) \tau$. In theory, the turbulence model of Treuhaft and Lanyi is a better way to describe tropospheric variations, but Jarlemark and Elgered (1998) showed empirically using water vapor radiometer (WVR) data that a random walk is the best fit for ZWD time series. We got similar results (cf. "ZWD noise characterization" section) and thus employ random walk processes for all ZWD estimations within the Kalman filter.

The time shift $\tau$ when calculating ASD of ZWD is chosen between the shortest possible duration (a few minutes for the KF depending on the spacing of the observation epochs and $20 \mathrm{~min}$ for LSM) and 1 day. The slope $k$ of a linear fit to the ASD values is calculated to determine the type of process noise. Then, assuming a random walk process and fixing $k$ to $-1 / 2$, the PSD $\Phi$ of the driving white noise is obtained.

For calculating the PSD of clock offsets, the procedure is similar. However, other than for ZWD, the ASD can only be computed for single days, even for continuous campaigns, as the time series has breaks due to the correlation in daily chunks. Further, it is of note that the clock offsets are always with respect to a reference clock. The obtained PSD is thus the sum of two clocks and accordingly, absolute station based values cannot be derived without external information. The investigated clock offsets are based on the main solutions, i.e., after quadratic polynomes from the first solutions have been subtracted.

The average ASD of the clock offsets from KF and LSM solutions for CONT14 is shown in Figs. 1 and 2, respectively, including those for individual days and stations (although w.r.t. a reference clock). The LSM clocks follow a random walk very closely $(k=-0.48$ when averaged over all CONT campaigns) and no contribution of an

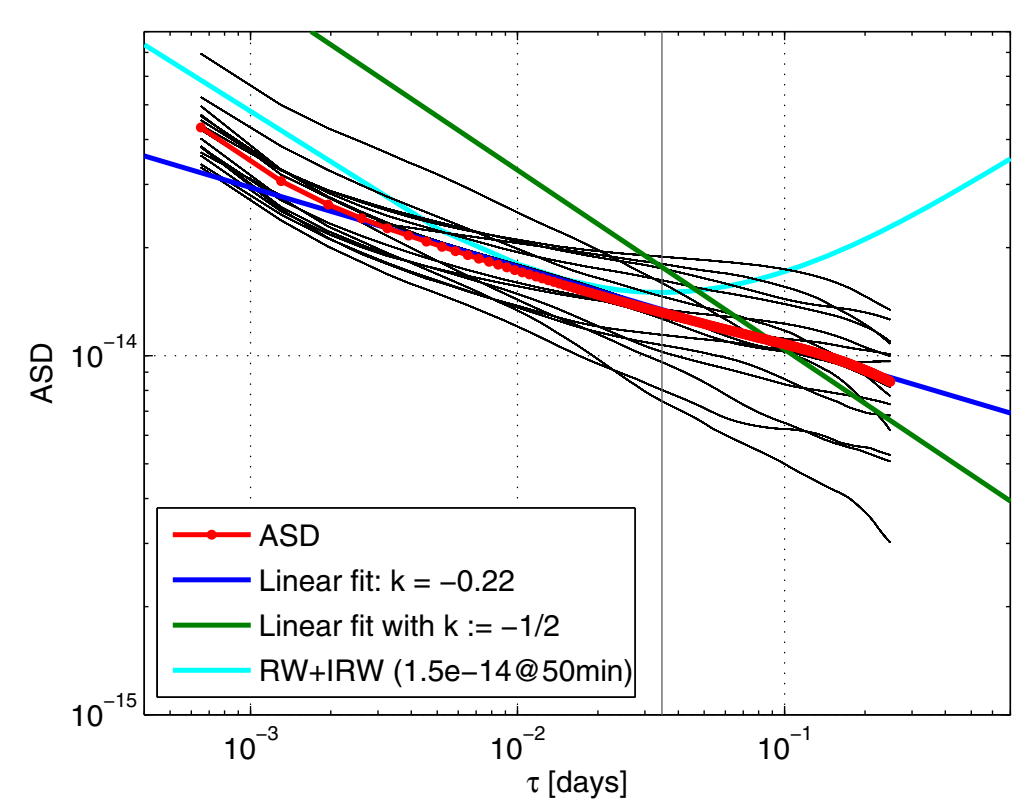

Fig. 1 Clock ASD for CONT14 from KF. Clock offset ASD values for time intervals between a few minutes and $6 \mathrm{~h}$ are shown logarithmically, based on a KF solution of CONT14. The individual values (see text) are represented as thin black lines, and the average is shown in red. Additionally, the plot includes a linear fit without constraints (blue) and one assuming a random walk process (green). The value $k$ represents the slope of the ASD in this log-log plot. The cyan curve corresponds to a theoretical clock model consisting of a random walk and an integrated random walk process. The vertical dark grey line marks 50 min 


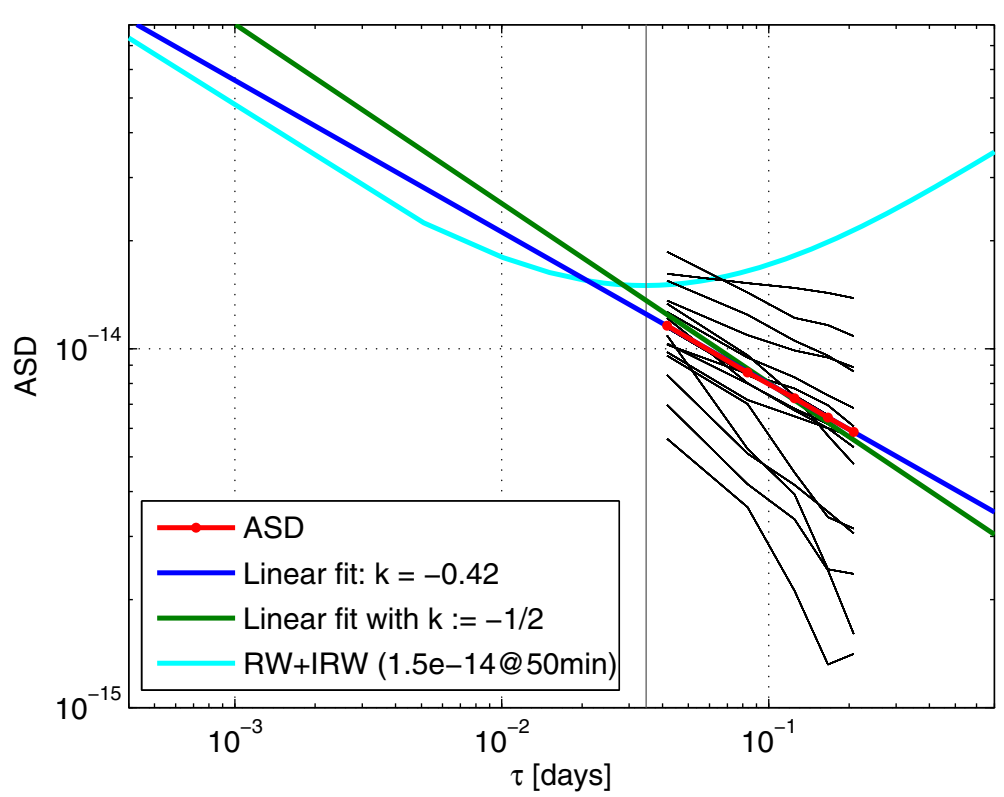

Fig. 2 Clock ASD for CONT14 from LSM. The same featues as in Fig. 1 are shown, with the clock offset data coming from LSM. Here, the minimum time shift is $1 \mathrm{~h}$ due to the coarser temporal resolution

integrated random walk can be detected, probably due to the removal of quadratic polynomes. The ASD from the KF solution is flatter, resembling a combination of random walk and integrated random walk processes up to about $50 \mathrm{~min}$. While the slope is different between KF and LSM, the noise level is similar at $\tau \approx 50 \mathrm{~min}$.

We used the ASD from LSM to estimate average PSD values for all CONT campaigns and found $\Phi$ to lie between 25 and $54 \mathrm{~cm}^{2} /$ day, corresponding to ASD of about 1 to $1.5 \cdot 10^{-14}$ for an interval of $50 \mathrm{~min}$. Conservatively, we applied the upper boundary as the default clock model in our KF solutions (cf. Table 1).

Table 1 Parameterizations of the KF and LSM solutions. For the KF part, the PSD $\Phi$ for random walk processes are given. For LSM, the temporal resolution of the piecewise linear functions estimated in the adjustment are given. The listed constraints are relative and valid for the intervals stated in the other column

\begin{tabular}{lrll}
\hline Parameter & \multicolumn{1}{c}{$\Phi(\mathrm{KF})$} & \multicolumn{2}{c}{ Interval (LSM) Constraints (LSM) } \\
\hline Station coordinates & $0.1 \mathrm{~cm}^{2} /$ day & 1 day & No constraints \\
Source coordinates & $0.01 \mathrm{mas}^{2} /$ day & 1 day & No constraints \\
Pole coordinates and UT1 & $0.1 \mathrm{mas}^{2} /$ day & 1 day & 0.0001 mas \\
Celestial pole offsets & $0.01 \mathrm{mas}^{2} /$ day & 1 day & 0.0001 mas \\
Clock parameters & $52 \mathrm{~cm}^{2} /$ day & $1 \mathrm{~h}$ & $1.3 \mathrm{~cm}$ \\
ZWD & $19 \mathrm{~cm}^{2} /$ day & $20 \mathrm{~min}$ & $1.5 \mathrm{~cm}$ \\
Gradients & $0.02 \mathrm{~cm}^{2} /$ day & $6 \mathrm{~h}$ & $0.05 \mathrm{~cm}$ \\
\hline
\end{tabular}

${ }^{a}$ The clock parameters are assumed to have an ASD of $1.5 \cdot 10^{-14}$ at $50 \mathrm{~min}$, corresponding to $\Phi=52 \mathrm{~cm}^{2} /$ day for a random walk

${ }^{b}$ The value given for ZWD is just an average as several different stochastic models for ZWD have been investigated within the scope of this study
In Herring et al. (1990), the VLBI delay rate observations were used to directly compute the PSD of the ZWD. However, other random walk processes affect the VLBI data as well, most notably station clocks. Herring et al. (1990) assumed that the clock noise is significantly smaller than that of the ZWD and would inflate the ZWD PSD estimates by less than $20 \%$. However, we found that the ZWD noise was on average lower than suggested by Herring et al. 1990 by a factor of about 3 (cf. the first part of the "Results and discussion" section) and thus smaller than that of the clocks (cf. Table 1). Other studies suggested that the ZWD noise might be even smaller, compared to Herring et al. (1990) by a factor of almost 10 (Schüler 2001). By neglecting the clock contribution, the ZWD PSD values would thus be strongly overestimated and therefore we decided not to follow this approach.

Two types of solutions were created using the Kalman filter. The first was derived by analyzing the VLBI data split into daily sessions, the other by feeding the data of the whole duration of an observation campaign continuously into the filter. The first type was needed to investigate baseline length and station coordinate repeatabilities, the second served as a reference of what the Kalman filter is capable of. The continuous solution has the advantage that no artificial jumps in the parameters occur at the daily session boundaries. The only exceptions are the clock offsets, for which jumps are allowed (a necessity due to the way VLBI data is currently correlated). The noise parameterization of the Kalman filter solutions can be found in Table 1. 
Primarily, the Kalman filter solutions were compared with the ones obtained from classical least squares adjustments. The LSM solutions were created using the estimation module "VIE_LSM" (Böhm et al. 2012) in VieVS@GFZ. Here, the VLBI data are always processed day by day and all parameters are modeled as piecewise linear functions. The temporal resolution and constraints for these functions are given in Table 1. The KF and LSM solutions used the same theoretical models for a priori correction of the observations. Furthermore, exactly the same observations were selected as outliers and also the choice of reference clocks and clock breaks was identical. The comparisons thus truly show the sole effect of different parameter estimation algorithms.

\section{Data}

The VLBI data were taken from the continuous "CONT" campaigns, organized by the International VLBI Service for Geodesy and Astrometry (IVS, Schuh and Behrend 2012). The CONT campaigns are outstanding compared to standard VLBI sessions by having observations scheduled continuously for 15 days, featuring a larger station network and recording data at a higher rate. As a result, the number of observations per day is larger than for normal 24-h VLBI sessions and thus the overall quality of results is better (e.g., Nilsson et al. 2014). Due to the efforts in organizing such campaigns, they have only been scheduled once every 3 years since 2002 . The data from CONT campaigns were chosen as the basis of our investigations as they represent the state-of-the-art capabilities of the VLBI technique and are closest to what is intended for VGOS. Characteristics of the individual CONT sessions are summarized in Table 2.

To estimate ZWD from VLBI data, first the zenith hydrostatic delays (ZHD) were calculated using the pressure records at the VLBI sites and the model of Saastamoinen (1972). The mapping functions VMF1
(Böhm and Schuh 2004) were used to project the slant delays to the zenith direction. The slant hydrostatic delays were removed before the adjustment. For gradients, a priori values provided by Böhm et al. (2013) were used.

Additionally, we computed station-specific ZWD values for CONT11 and CONT14, utilizing numerical weather model (NWM) data and the ray tracing (RT) algorithm described by Zus et al. (2012, 2014). For comparison, two different NWM were used, the Global Forecast System (GFS) analysis of the National Centers for Environmental Prediction (NCEP) and the Integrated Forecast System (IFS) analysis of the European Centre for Medium-Range Weather Forecasts (ECMWF). The GFS analysis is available every $6 \mathrm{~h}$ with horizontal resolutions of $1^{\circ}$ and $0.5^{\circ}$ on 26 pressure levels. In addition, short-range forecasts based on the previous analysis epoch are available, offset by $3 \mathrm{~h}$. By combining the analysis and forecast data, a temporal resolution of $3 \mathrm{~h}$ for the meteorological data is possible. We tested both spatial $\left(1^{\circ} / 0.5^{\circ}\right)$ and temporal resolutions $(6 \mathrm{~h} / 3 \mathrm{~h})$ and found the $0.5^{\circ} / 3 \mathrm{~h}$ setup performing best. The IFS analysis is available every $6 \mathrm{~h}$ with a horizontal resolution of $1^{\circ}$ on 137 model levels.

With a WVR, the wet atmospheric delay can be inferred from measurements of the thermal radiation from the sky at a few frequencies. Typically two frequencies are used, one close to the 22- $\mathrm{GHz}$ water vapor line and the other around $30 \mathrm{GHz}$, where the sensitivity to liquid water is higher. By combining the measurements of the sky brightness temperatures at two frequencies, the contribution from water vapor and liquid water can be separated. The water vapor part can then be converted into a wet delay using an empirical retrieval factor. For more details, see, e.g., Elgered (1993) or Nilsson et al. (2013).

A few VLBI stations operate WVR that can be used for the comparison of tropospheric parameters. For CONT11, we obtained data from the two radiometers in Onsala, Astrid (Elgered and Jarlemark 1998), and Konrad

Table 2 Comparison of VLBI CONT campaigns. During CONT11, 14 stations participated, but one of them, Warkworth, New Zealand, only observed for a few hours and was therefore excluded from the analysis. The number of observation epochs given below is equivalent to the number of state updates performed in the KF. Usually, only one scan (i.e., the observation of a particular radio source) is scheduled at a time, but in some cases sub-networks perform different scans at the same time. Thus, the number of observation epochs is slightly lower compared to the number of scans. The numbers take into account that some observations flagged as outliers were removed. In the correlation process, the CONT campaigns are split into sessions lasting $24 \mathrm{~h}$ each. These start at 18:00 for CONT02, 17:00 for CONT05, and 0:00 for the others

\begin{tabular}{lrrrr}
\hline Campaign & Date & $\begin{array}{r}\text { Number of } \\
\text { stations }\end{array}$ & $\begin{array}{r}\text { Recording } \\
\text { rate (MBit/s) }\end{array}$ & $\begin{array}{r}\text { No. of } \\
\text { observation epochs }\end{array}$ \\
\hline CONT02 & October 16-31, 2002 & 8 & 128 & 6911 \\
CONT05 & September 12-27, 2005 & 11 & 256 & 12,879 \\
CONT08 & August 12-26, 2008 & 11 & 512 & 17,215 \\
CONT11 & September 15-29, 2011 & 13 & 512 & 16,215 \\
CONT14 & May 6-20, 2014 & 17 & 512 & 22,915 \\
\hline
\end{tabular}


(Stoew and Rieck 1999), as well as from the Radiometrix radiometer in Tsukuba. For CONT14, we only had data from the Konrad radiometer in Onsala. The Tsukuba radiometer was only measuring in the zenith direction, while the Onsala radiometers were operated in a "sky mapping mode", resulting in a good sky coverage above $20^{\circ}$ elevation angle. Lower elevation angles are not possible in order to avoid picking up radiation from the ground. Since WVR do not work well during rain, the data from rainy periods were removed by eliminating all data points where the estimated atmospheric liquid water content was above $0.7 \mathrm{~mm}$, a strong indication for rain.

The GPS data, used for comparison and validation, were processed with GFZ's GNSS analysis software package, Earth Parameter and Orbit determination System (EPOS), which is used to contribute to International GNSS Service (IGS) activities as one of the leading IGS Analysis Centers (Gendt et al. 1999).

The GPS results for CONT11 are based on the GFZ contribution to the IGS Tide Gauge Benchmark Monitoring (TIGA) reprocessing project. GFZ is one of the TIGA Analysis Centers (Schöne et al. 2009) and homogenously reprocessed GPS data acquired from 794 globally distributed sites, covering the time period from January 1994 to December 2012. In this solution, the a priori tropospheric delays were obtained using the model by Saastamoinen, the current Global Pressure and Temperature model (GPT2, Lagler et al. 2013), and the mapping functions VMF1. The zenith total delays (ZTD) were estimated together with the horizontal delay gradients using an elevation cut-off angle of $7^{\circ}$. The ZTD are provided every hour while gradients are given once per day. More information about the solution can be found in Deng et al. (2015). During CONT11, the VLBI collocated GPS stations ONSA and TSKB are included in the comparisons.

For CONT14, only ONSA is used for the validation of the results of the other techniques. Here, the GPS solution is based on the operational GPS data processing at GFZ using precise point positioning (PPP), supplying several tropospheric products and contributing to various meteorological projects (Dick et al. 2000, 2001; Gendt et al. 2004). In a first step, high-quality GPS orbits and clocks were estimated from a global network of about 50 IGS sites. Secondly, parameters like ZTD were derived using PPP with the orbits and clocks fixed to those from the first step. The same cut-off angle and models related to the troposphere as in the TIGA reprocessing were used, and both the ZTD and the gradients were estimated once per hour. Preliminary tests with GPS data of higher temporal resolution have not led to significant changes in the results of the comparisons.

The GPS solutions provide ZTD values, which need to be converted to ZWD. First, the pressure at the GPS sites was calculated using the local pressure measurements at the collocated VLBI sites and corrected for the height difference by applying the model by Hopfield (1969). Next, the model by Saastamoinen was used to calculate ZHD, which were subtracted from the ZTD, yielding ZWD.

\section{Results and discussion \\ ZWD noise characterization}

To determine the PSD $\Phi$, ZWD time series were derived for all five CONT campaigns and all participating stations, using both the KF and LSM approach. For the initial KF solution, a PSD of $\Phi=56 \mathrm{~cm}^{2} /$ day, taken from Herring et al. (1990), was used. This value is larger than others from literature (e.g., $\Phi=6 \mathrm{~cm}^{2} /$ day, Schüler 2001) and thus gives the observations more weight compared to the predictions. Tests with different $\Phi$ values showed that a larger initial $\Phi$ value does not affect the estimated PSD as much as smaller ones. The approach to estimate $\Phi$ via the Allan standard deviation was followed as described in the "Methods" section.

Figure 3, as an example, shows the ASD for the fundamental station in Wettzell, Germany, during CONT14. The difference between the estimated slope and the value $-1 / 2$ can be seen as an indicator whether the assumption of a random walk is warranted and thus also for the quality of the estimated $\Phi$ values. All following $\Phi$ values were obtained by fixing $k$ to $-1 / 2$. Table 3 lists $\Phi$ and $k$ values for every CONT campaign, averaged over all VLBI stations, for both the KF and LSM solution. While it is not surprising that the $k$ values of the KF solution are close to that of a random walk, also the results from LSM indicate that the tropospheric delays can be better described by a random walk than using turbulence theory $(k=-2 / 3)$. On average, the slope $k$ is slightly steeper for LSM, resulting in larger $\Phi$ values when $k$ is set to $-1 / 2$. The difference in $\Phi$ from KF and LSM amounts to $12 \%$ on average. All derived values are well within the boundaries indicated by literature (see above, 6 to $56 \mathrm{~cm}^{2} /$ day, Herring et al. 1990; Schüler 2001).

To validate the VLBI results with external data, the same procedure was repeated for the ZWD time series from WVR, GPS, and RT. Here, only the stations where all techniques were available for comparison were considered, i.e., Onsala, Sweden, and Tsukuba, Japan, for CONT11 and Onsala for CONT14. The results are shown in Table 4. A good agreement among the different techniques is found, with all supporting smaller $\Phi$ values for Onsala compared to Tsukuba during CONT11. The values for Onsala are very different between CONT11 and CONT14 for all techniques and amount to 28.1 and $6.5 \mathrm{~cm}^{2} /$ day, respectively, when averaged. The differences are probably due to different weathers at the time the observations were performed, related to the fact that CONT11 happened during 


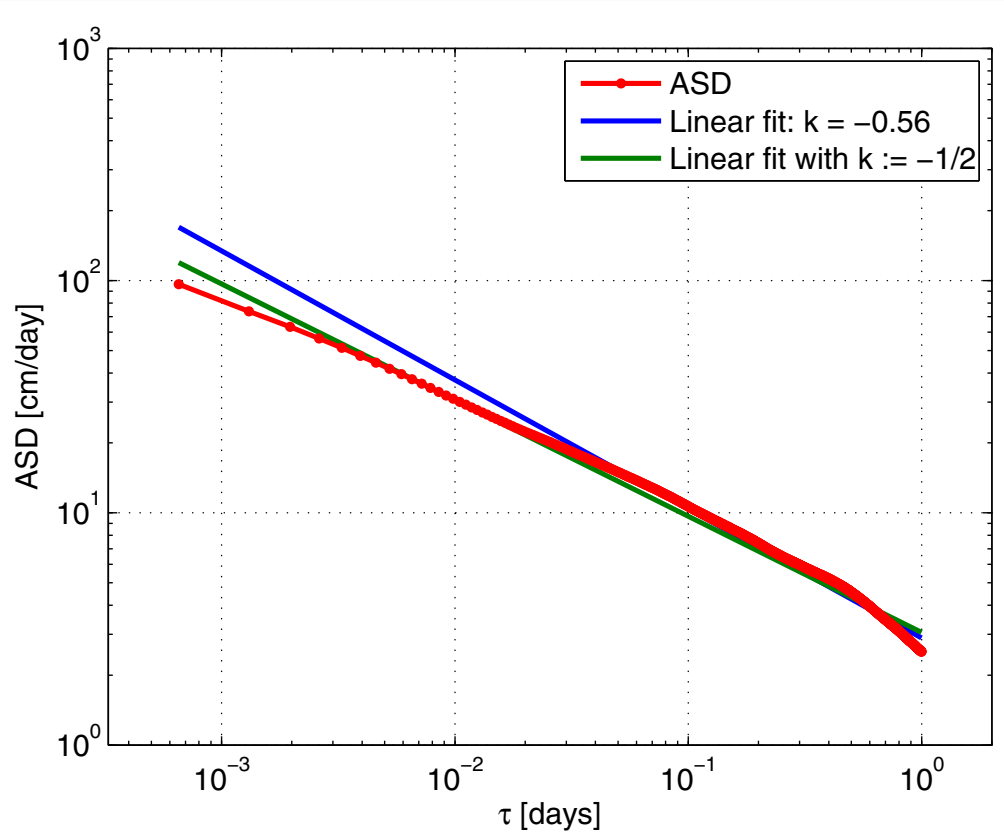

Fig. 3 ZWD ASD for Wettzell during CONT14. For the station Wettzell, Germany, ASD for time intervals between a few minutes and 1 day are shown logarithmically. The underlying ZWD time series is a first KF solution of CONT14. Additionally, the plot includes a linear fit without constraints (blue) and one assuming a random walk process (green). The value $k$ represents the slope of the ASD in this log-log plot

September and CONT14 during May. The PSD for Onsala during CONT11 agrees well with $\Phi=25.1 \mathrm{~cm}^{2} /$ day, which was used by Jarlemark (1997). In Jarlemark et al. (1998), values of 15.6 and $18.1 \mathrm{~cm}^{2} /$ day were obtained for Onsala what is close to the average of the values for CONT11 and CONT14. The RT results are provided only for NCEP and they should be taken with care; the low temporal resolution only allows the computation of very few ASD values and consequently, the estimation of $\Phi$ and $k$ is barely redundant. For $\Phi$, the KF solution for Onsala, CONT11, shows a better agreement with the other techniques, while both KF and LSM solutions have a similar difference to the average process noise for Tsukuba, CONT11, although with different signs. For CONT14, the

Table 3 Power spectral densities and noise characterizations for CONT campaigns. For both KF and LSM ZWD time series, the PSD $\Phi$ in $\mathrm{cm}^{2}$ /day, obtained by assuming a random walk process and fitting the ASD data accordingly, are shown. Additionally, the slope $k$ of the ASD fit is shown, indicating the type of noise

\begin{tabular}{lllll}
\hline Data set & $\Phi_{\mathrm{KF}}$ & $k_{\mathrm{KF}}$ & $\Phi_{\mathrm{LSM}}$ & $k_{\mathrm{LSM}}$ \\
\hline CONT02 & 12.7 & -0.44 & 14.8 & -0.53 \\
CONT05 & 23.9 & -0.45 & 27.7 & -0.54 \\
CONT08 & 22.8 & -0.48 & 25.3 & -0.55 \\
CONT11 & 19.8 & -0.48 & 23.7 & -0.58 \\
CONT14 & 15.6 & -0.49 & 17.2 & -0.56 \\
\hline Average & 19.0 & -0.47 & 21.7 & -0.55 \\
\hline
\end{tabular}

KF solutions agrees better with GPS and RT and the LSM solution with WVR data. The $k$ values vary between -0.38 (KF and GPS, Tsukuba, CONT11) and -0.73 (RT, Onsala, CONT14). The slope $k$ is generally steeper for Onsala compared to Tsukuba. Regarding $k$, the KF solution fits better to that of GPS, while the LSM solution is closer to the WVR data.

As the ZWD stochastic models obtained from KF and LSM time series are similar, for the rest of the paper we only use the KF one. Through the comparison with other techniques, it has not become clear which of the two is preferable, but because the slope $k$ of the KF solution is on average closer to $-1 / 2$, the errors due to the assumption of a random walk should be smaller compared to LSM. Station-based values of $\Phi$, averaged over all CONT campaigns, can be found in Fig. 4, showing color-coded PSD values on a world map. The numerical values are provided in Table 5 and used as default in the Kalman filter of VieVS@GFZ. It becomes evident that locations which are known for strong weather phenomena like Westford, United States, and Tsukuba, Japan, show very large noise parameters, confirming results by Nilsson and Haas (2010). In general, stations closer to the sea show larger $\Phi$ values compared to continental stations. Additionally, stations closer to the equator have a tendency of larger noise parameters compared to those closer to the poles. An excellent example for this is Ny-Ålesund, Norway, being located at a latitude of $79^{\circ}$ and featuring the lowest value $\Phi=3.1 \mathrm{~cm}^{2} /$ day. 
Table 4 ZWD noise characterization using different observation techniques. Given are PSD values and ASD slopes from different observing techniques for selected stations (cf. Table 3). The PSD $\Phi$ is given in $\mathrm{cm}^{2} /$ day

\begin{tabular}{|c|c|c|c|c|c|c|c|c|c|c|}
\hline Data set & $\Phi_{\mathrm{KF}}$ & $k_{\mathrm{KF}}$ & $\Phi_{L S M}$ & $k_{L S M}$ & $\Phi_{\text {WVR }}$ & $k_{W V R}$ & $\Phi_{\mathrm{GPS}}$ & $k_{\mathrm{GPS}}$ & $\Phi_{R T}$ & $k_{\text {RT }}$ \\
\hline Onsala, CONT11 & 28.0 & -0.46 & 30.1 & -0.54 & 24.0 & -0.55 & 27.5 & -0.46 & 30.9 & -0.57 \\
\hline Tsukuba, CONT11 & 39.8 & -0.38 & 53.8 & -0.52 & 47.1 & -0.51 & 44.2 & -0.38 & 49.9 & -0.44 \\
\hline Onsala, CONT14 & 6.0 & -0.55 & 6.5 & -0.63 & 8.1 & -0.69 & 6.0 & -0.54 & 5.7 & -0.73 \\
\hline
\end{tabular}

Some of the VLBI stations have performed in several CONT campaigns. The PSD values of stations that have participated in three or more CONT campaigns are plotted in Fig. 5. Additionally, the averaged $\Phi$ value for every CONT campaign is shown. The largest variations over time are found for stations Westford and Tsukuba, which feature the largest average $\Phi$ values. A pronounced change in $\Phi$ is also found for Onsala, between CONT11 and CONT14. Some of the variations for particular stations might be explained by different weather during each CONT campaign and the different seasons in which they took place. Still, with the exception of CONT02, which is strongly affected by the small $\Phi$ value for Westford, the magnitude of ZWD variations has decreased continuously. The mean $\Phi$ values are of course strongly affected by the changes in the observing network, but it might be a hint that the quality of VLBI measurements has increased in the past years, causing less artificial noise in the ZWD time series. For any climatic interpretations, however, the time frame is too short.

\section{Effects on station coordinates}

It is known that errors in tropospheric parameters propagate to the estimates of station coordinates (Nilsson et al. 2013). With a better handling of the tropospheric delays, improvements in the quality of derived station coordinates are expected. Widely used measures to quantify the effects on station positions are station coordinate and baseline length repeatabilities (Davis et al. 1985). Nilsson et al. (2015) showed that by using a Kalman filter instead of least squares adjustment, an improvement in the baseline length repeatabilities of about $10 \%$ is possible. Here, we wanted to focus on the effect of a station-specific noise model for ZWD.

For this purpose, we used the Kalman filter solution which is based on the CONT campaigns split into daily segments. For every day, average station positions were derived and the lengths of all possible baseline were calculated. The baseline length repeatabilities were then computed as the WRMS of the daily baseline lengths. For every CONT campaign, the specific station-based noise models discussed in the previous section were applied and the solutions were compared to those using the average ("global") values for each CONT campaign as found in Table $3\left(\Phi_{\mathrm{KF}}\right)$.

Figure 6 shows the baseline length repeatabilities plotted against the length of the baselines for CONT14. A function comprising a quadratic and constant term was fit to the two solutions, and the repeatabilities for a theoretical baseline length of 10,000 km were computed. The results for all CONT campaigns are shown in Table 6. For every single campaign, the station-based ZWD noise model performs better; on average, the WRMS is reduced by $2.3 \%$, and $75 \%$ of the baselines are improved. All percentages in this work related to improvements in terms of standard deviations or RMS are calculated by

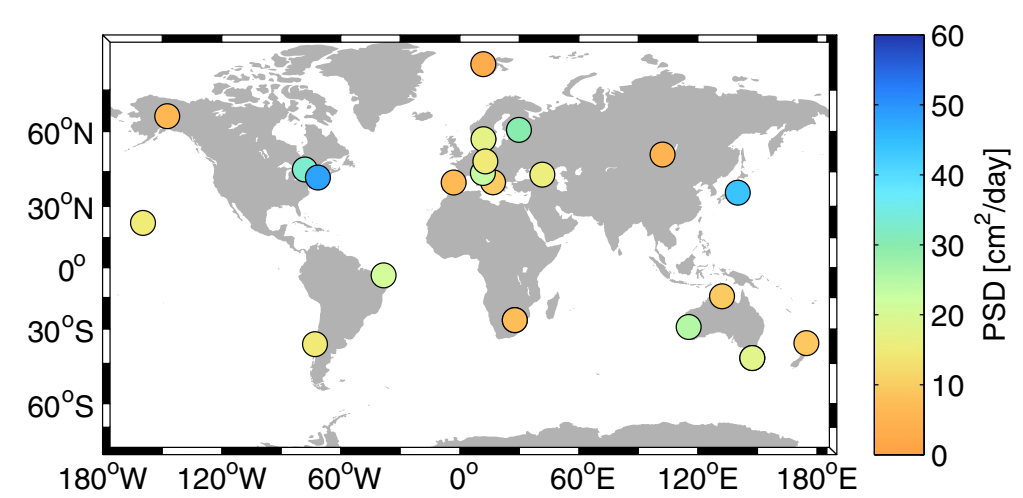

Fig. 4 ZWD PSD map averaged over all CONT campaigns. The average PSD from all CONT campaigns is shown color coded for every participating station. The PSD values in this map have been averaged from the KF solutions of the individual CONT campaigns. The exact values can be found in Table 5 
Table 5 Station-based PSD model for ZWD. For all stations that participated in at least one of the CONT campaigns, the average PSD $\Phi$, given in $\mathrm{cm}^{2} /$ day and obtained from all KF ZWD time series, are shown together with the average ASD slopes $k$. Figure 4 is a graphical representation of these values

\begin{tabular}{|c|c|c|}
\hline IVS station name & $\Phi_{\mathrm{KF}}$ & $k_{\mathrm{KF}}$ \\
\hline ALGOPARK & 33.1 & -0.34 \\
\hline BADARY & 5.0 & -0.49 \\
\hline FORTLEZA & 21.2 & -0.56 \\
\hline GILCREEK & 5.9 & -0.48 \\
\hline HOBART12 & 18.0 & -0.46 \\
\hline HARTRAO & 8.9 & -0.48 \\
\hline HOBART26 & 18.4 & -0.45 \\
\hline HART15M & 7.4 & -0.48 \\
\hline KATH12M & 9.8 & -0.54 \\
\hline KOKEE & 14.8 & -0.53 \\
\hline MATERA & 9.9 & -0.52 \\
\hline MEDICINA & 23.4 & -0.56 \\
\hline NYALES20 & 3.1 & -0.52 \\
\hline ONSALA60 & 17.5 & -0.46 \\
\hline SVETLOE & 30.1 & -0.40 \\
\hline TIGOCONC & 14.6 & -0.43 \\
\hline TSUKUB32 & 44.0 & -0.38 \\
\hline WESTFORD & 48.2 & -0.35 \\
\hline WARK12M & 9.3 & -0.52 \\
\hline WETTZELL & 14.7 & -0.45 \\
\hline YARRA12M & 25.3 & -0.41 \\
\hline YEBES40M & 6.8 & -0.55 \\
\hline ZELENCHK & 15.9 & -0.65 \\
\hline
\end{tabular}

dividing the difference of two quantities by one of the quantities.

Furthermore, we compared the effect of using the CONT-specific station-based noise model versus the average station-based model that can be found in Table 5 and Fig. 4. Here, the differences in the baseline length repeatabilities are less pronounced. For CONT02, CONT08, and CONT11, the specific station-based models result in slightly better repeatabilities (improvements by $0.9 \%$ on average). CONT14 is basically not affected with a difference of just $0.1 \%$. This is reasonable, as the model derived only from CONT14 data is very similar to the one utilizing data from all CONT campaigns. For CONT05, the average station-based model performs better by $0.2 \%$, which might be related to the unusual PSD values for Westford and Tsukuba during CONT05 compared to their average ones $\left(28.9\right.$ vs. $48.2 \mathrm{~cm}^{2} /$ day for Tsukuba and 80.2 vs. $44.0 \mathrm{~cm}^{2} /$ day for Westford). Averaged over all CONT sessions, the improvement gained by using models created from a specific CONT campaign $(0.5 \%)$ is thus less pronounced than by using a station-specific model compared to a globally constant one $(2.3 \%)$.

When investigating station coordinate repeatabilities, the results are very similar compared to baseline length repeatabilities. As an example, Fig. 7 shows the 3D coordinate repeatabilities for CONT14 based on the KF time series with station-based and average ZWD noise as well as the LSM solution. Similar to baseline lengths, the station-based KF solution performs best for all CONT campaigns (Table 7). For 3D coordinate repeatabilities, the improvement is a exactly the same ( $2.3 \%$ on average). In addition to the $3 \mathrm{D}$ repeatabilities, Fig. 8 includes the repeatabilities of the individual components. Here, the difference in WRMS for the KF solutions using station-based and global ZWD noise is shown for every CONT campaign, averaged over all participating stations. It becomes evident that the largest improvement is found in the height component, which is known to feature a higher correlation to ZWD than the horizontal coordinates. The worse repeatabilities for the horizontal components during CONT11 are related to two individual stations: Zelenchukskaya for the east component and Yebes for the north component. For both instances, the repeatability is worse for the station-based noise model by a factor of about 2. This might be related to the fact that for both stations, the assumption of a random walk (i.e., ASD proportional to $\tau^{-0.5}$ ) when computing the stationbased PSD values is not ideal with exponents of -0.68 and -0.59 , respectively. When using the median of the repeatabilities instead of the mean, the station-dependent noise model is favorable also for the horizontal components during CONT11, which indicates that this problem is related only to the two stations.

\section{Comparison of ZWD from different techniques}

In this section, the VLBI ZWD time series obtained using KF and LSM are compared to those from other techniques described in the "Data" section. Due to the limited availability of the WVR data, the comparisons are only performed for the stations Onsala and Tsukuba in CONT11 and Onsala in CONT14. Here, the KF solutions for which the CONT campaigns are processed as a whole are used. Although the comparisons have been conducted including both stochastically station-specific and global KF solutions, all plots in this section only show the station-based one due to their similarity.

Figure 9 shows the ZWD time series for station Onsala during CONT11, with different colors representing the various techniques. The deficiencies of the ray-traced delays due to the limited temporal resolution become apparent immediately. Both data sets fail to capture the level of detail of the other techniques, which is in 


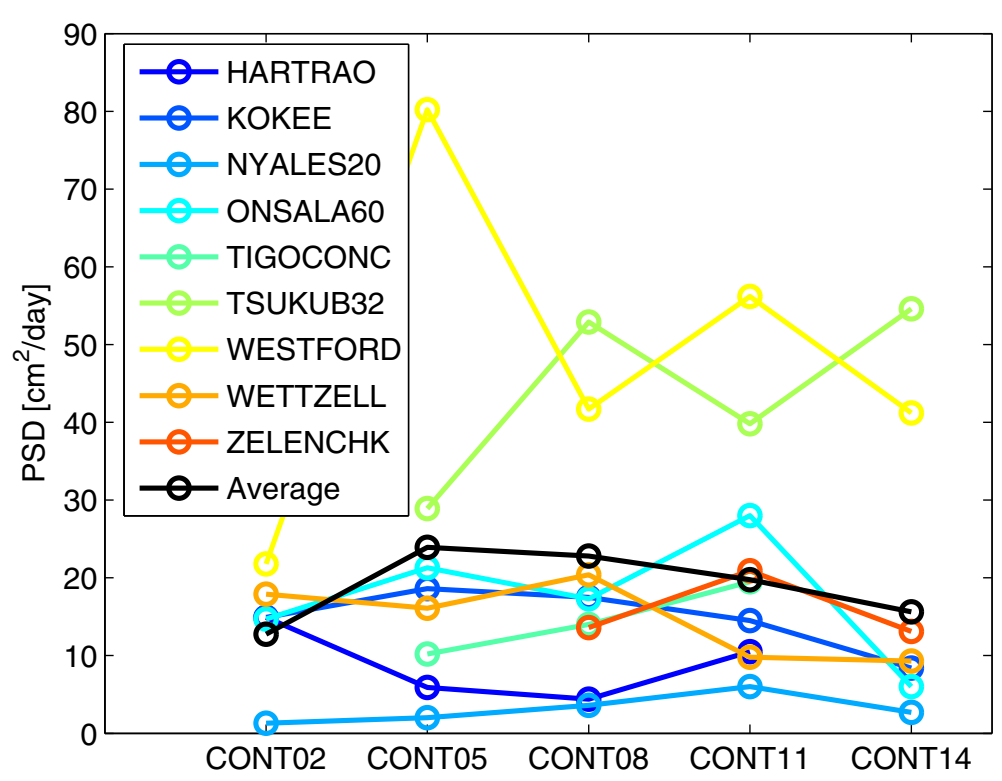

Fig. 5 ZWD PSD time series based on the CONT campaigns. For every station that participated three or more times in CONT campaigns, the PSD values from the KF ZWD time series are shown. Additionally, the average PSD value of each CONT campaign is included (black)

particular notable for peaks in the time series. The NCEP data, provided at three-hourly intervals, performs better in this regard. In this plot, it also becomes evident that the WVR data has gaps, as observations during rainfall events have been eliminated. A plot for Onsala during CONT14 would be very similar to Fig. 9 and is thus not included, although the WVR data is slightly noisier.

In Fig. 10, the different time series for Tsukuba are plotted, but instead of covering the whole duration of CONT11, only 2 days are shown. This allows for a better

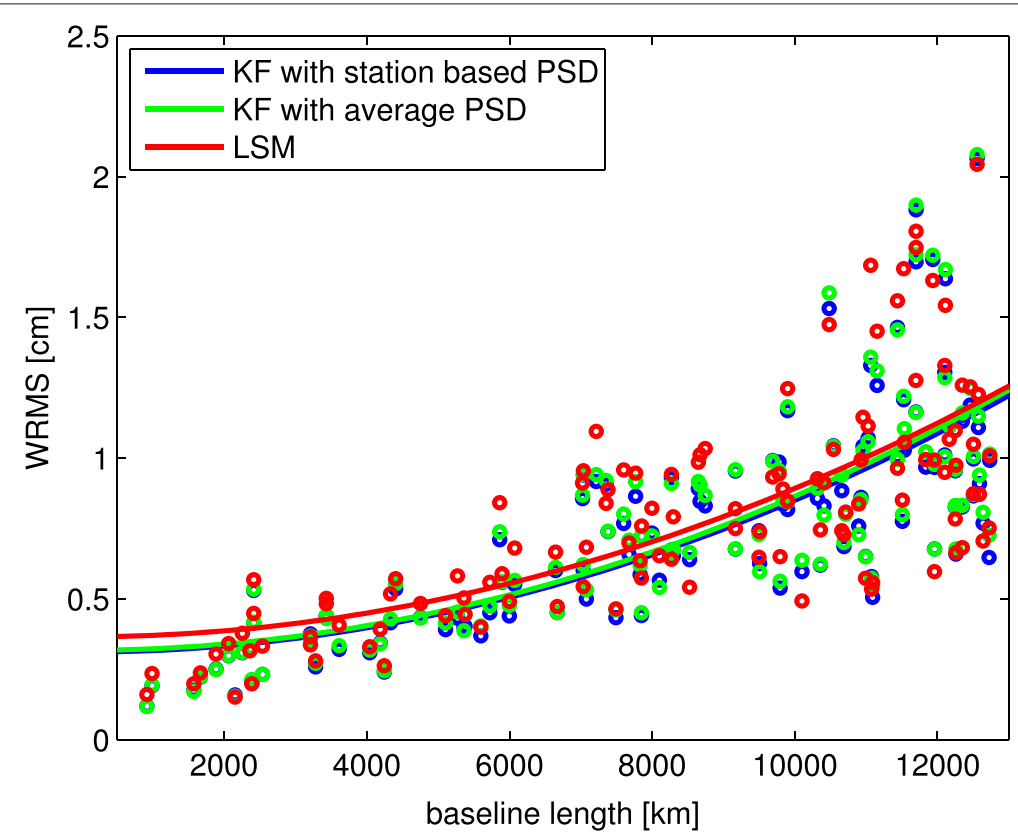

Fig. 6 Baseline length repeatabilities for CONT14. Shown are baseline length repeatabilities for CONT14, based on a KF solution with station-dependent ZWD noise parameters (blue) and on one with the same PSD for all stations (green), as a function of baseline length. Additionally, a reference solution from LSM is plotted (red). Every triple of blue, green, and red dots represents a single baseline. The curves are quadratic fits based on all available baselines 
Table 6 Baseline length repeatabilities. Given are baseline length repeatabilities for a KF solution using a global $\Phi$ value and one with station-dependent $\Phi$. Both types of $\Phi$ values have been extracted from time series of the specific CONT campaigns. The repeatabilities are valid for a theoretical baseline of 10,000 km, calculated from a quadratic fit of the individual baseline length repeatabilities

\begin{tabular}{llllc}
\hline Campaign & \multicolumn{2}{c}{ Repeatabilies (mm) } & Improvement (\%) & $\begin{array}{c}\text { Fraction of } \\
\text { improved stations (\%) }\end{array}$ \\
\cline { 2 - 5 } \multicolumn{2}{l}{ Station based } & Global & & 5.2 \\
CONT02 & 14.25 & 15.04 & 1.8 & 73 \\
CONT05 & 12.30 & 12.53 & 2.4 & 78 \\
CONT08 & 9.33 & 9.56 & 0.3 & 60 \\
CONT11 & 12.24 & 12.28 & 1.6 & 68 \\
CONT14 & 8.50 & 8.64 & & \\
\hline
\end{tabular}

discernability of the individual time series, making the small differences between the KF and LSM VLBI solutions detectable, for example, at $t \approx 14.2$ days. Furthermore, it becomes evident that VLBI and GPS data agree well and both are offsets against the WVR data by more than $2 \mathrm{~cm}$.

Out of the techniques included in this study, WVR is the most direct to measure the wet delays of the atmosphere and also provides the highest temporal resolution. Therefore, we selected this data set as the reference. In order to calculate differences, the time series of the other techniques were linearly interpolated to match the temporal resolution of the WVR data. At epochs, for which no WVR data are available, also the data of other techniques were eliminated. Then, the WVR values were subtracted from all other data sets, and biases, RMS values, and standard deviations (RMS after removing the biases) were calculated. The results are found in Table 8. As an example, Fig. 11 shows the differences w.r.t. WVR for Onsala during CONT14. To make the plot less noisy (the WVR in this case provides ZWD values every 6 s), the differences were smoothed with a moving average filter using a window size of $50 \mathrm{~min}$. For the results found in Table 8, no smoothing was applied.

For Onsala, the biases w.r.t. WVR are less than $1 \mathrm{~cm}$ for all techniques. The WVR in Tsukuba, however, does not appear to be calibrated well, resulting in offsets larger than $2 \mathrm{~cm}$. In all three study cases, a different VLBI or GPS solution is on average closer to WVR. Smaller biases are found for the RT solutions. However, due to the calibration issues, WVR is probably not an ideal reference for the average ZWD and related considerations have to be taken with care. When comparing NCEP and ECMWF biases, the latter is always closer to GPS and VLBI. Ray-traced NCEP data seem to overestimate the ZWD by a few millimeters compared to ECMWF. The average ZWD from GPS and VLBI agrees very well, during CONT11 by less than $0.5 \mathrm{~mm}$ and by $2 \mathrm{~mm}$ during CONT14. The KF and LSM solutions have differences in the biases of $0.1 \mathrm{~mm}$ for

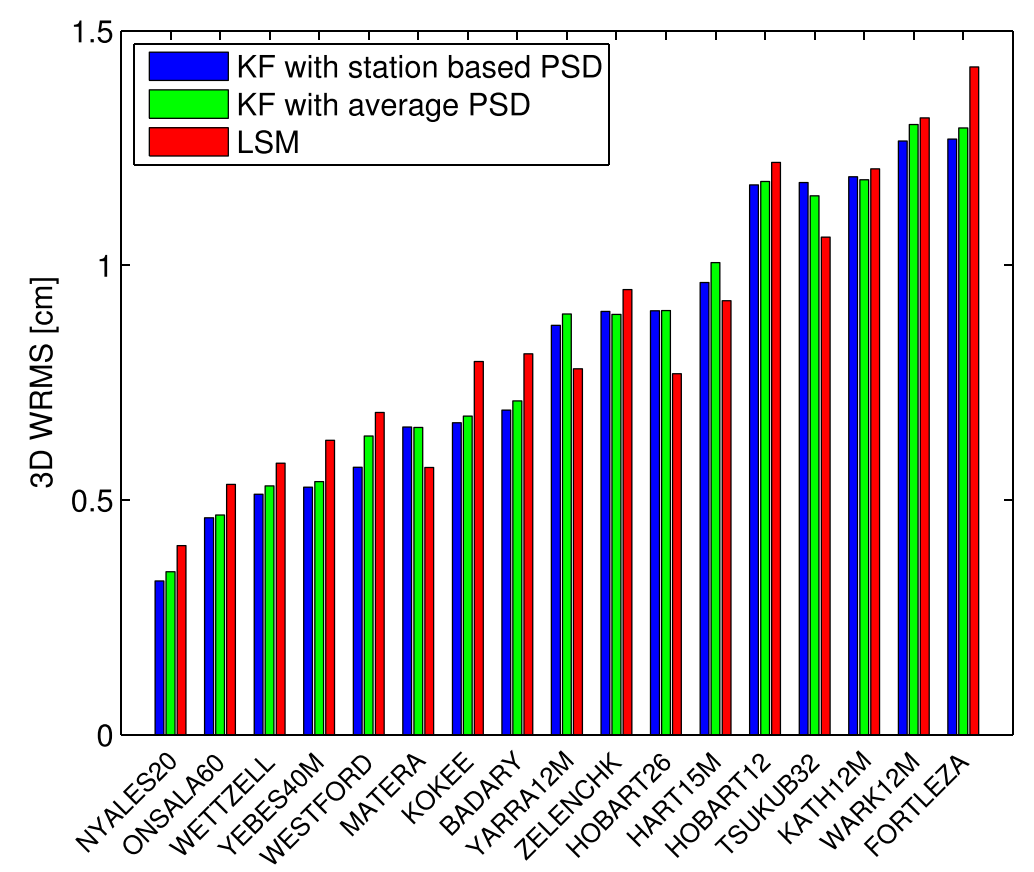

Fig. 7 3D position repeatabilities for CONT14. Shown are 3D station coordinate repeatabilities for CONT14, based on a KF solution with station-dependent ZWD noise parameters (blue) and on one with the same PSD for all stations (green), for every participating station. The stations are sorted by their WRMS values for the station dependent solution. Additionally, a reference solution from LSM is plotted (red) 
Table 7 Station coordinate repeatabilities. Given are station coordinate repeatabilities for a KF solution using a global $\Phi$ value and one with station-dependent $\Phi$. Both types of $\Phi$ values have been extracted from time series of the specific CONT campaigns. Here, the repeatabilities are defined as the WRMS of the 3D position variations

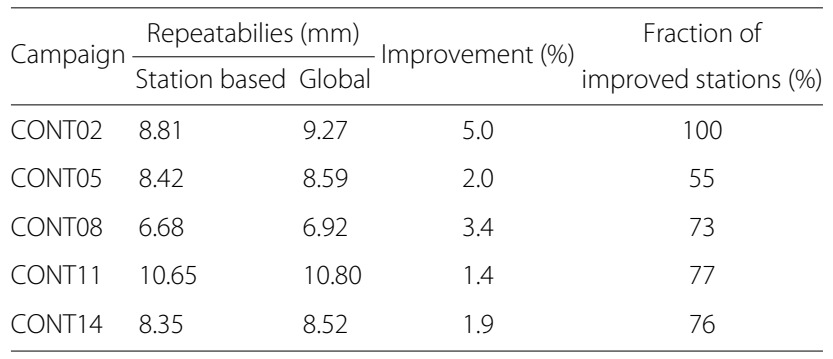

Onsala and at the sub-millimeter level for Tsukuba. This excellent agreement is not surprising as the expectation value of the Kalman filter should in theory be identical to the one of a least squares adjustment.

As the RMS is strongly affected by the biases mentioned in the last paragraph, the standard deviation (STD) with respect to WVR is analyzed in greater detail. In all cases, the RT data performs worst which is expected due to the inferior temporal resolution, with STD values sometimes twice as large compared to the other solutions. During CONT11, the STD of the GPS solution is up to $0.3 \mathrm{~mm}$ smaller than the best VLBI solution, even though the temporal resolution of $1 \mathrm{~h}$ is worse than that of the KF and LSM solutions. During CONT14, the VLBI KF solution performs slightly better than GPS. When comparing the two VLBI solutions, it is evident that the KF is able to outperform LSM in all three cases. The improvement is $15 \%$ for CONT11 and $6 \%$ for CONT14. This is most likely due to the better temporal resolution of the KF. The difference in STD between the KF solutions with station-wise tuned and global stochastic models lies between 0.3 and $0.8 \%$ in favor of the former for all three cases.

Spectra of the differences were investigated as well, using Lomb-Scargle periodograms, but only large amplitudes for periods of fractals of 15 days, the duration of the CONT campaigns, were detected. Other than these artificial signals, only daily signals, although much weaker, were found. In the case of VLBI, these signals could possibly be related to the splitting of the CONT campaigns into daily sessions.

Finally, the formal errors of the tropospheric delays provided by different observation techniques were compared for selected stations. For VLBI and WVR, ZWD formal errors are available, for GPS only those of ZTD. The difference in the formal errors of ZTD and ZWD depends on the precision of the pressure data used to correct for ZHD. In VLBI analysis, the pressure data is assumed as perfectly known and therefore the a priori standard deviations of the group delays are not adjusted when the hydrostatic delays are subtracted. To be consistent, we have to assume the same for the GPS data (as we use the

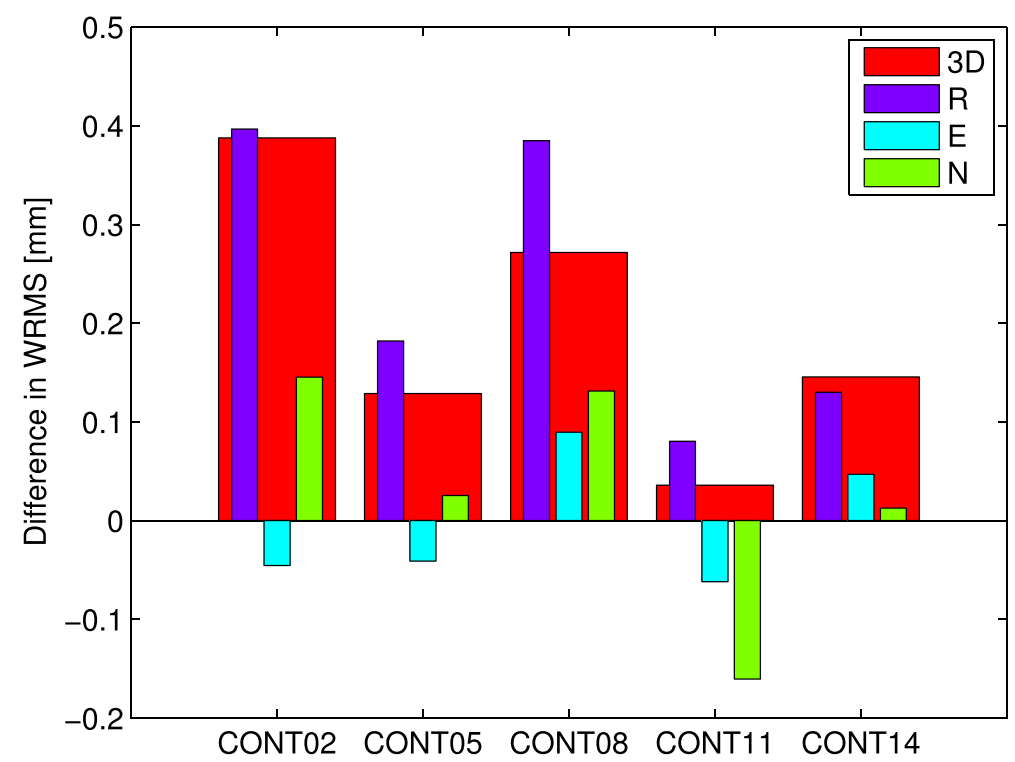

Fig. 8 Differences in station coordinate repeatabilities for the CONT campaigns. For every CONT campaign, the difference in repeatabilities of a KF solution with station-dependent ZWD noise parameters and one with the same PSD for all stations are shown. Positive values indicate that the station-dependent model performs better. The repeatabilities are given for the radial $(R)$, east $(E)$, and north $(N)$ components of the station coordinates, as well as for the 3D positions 


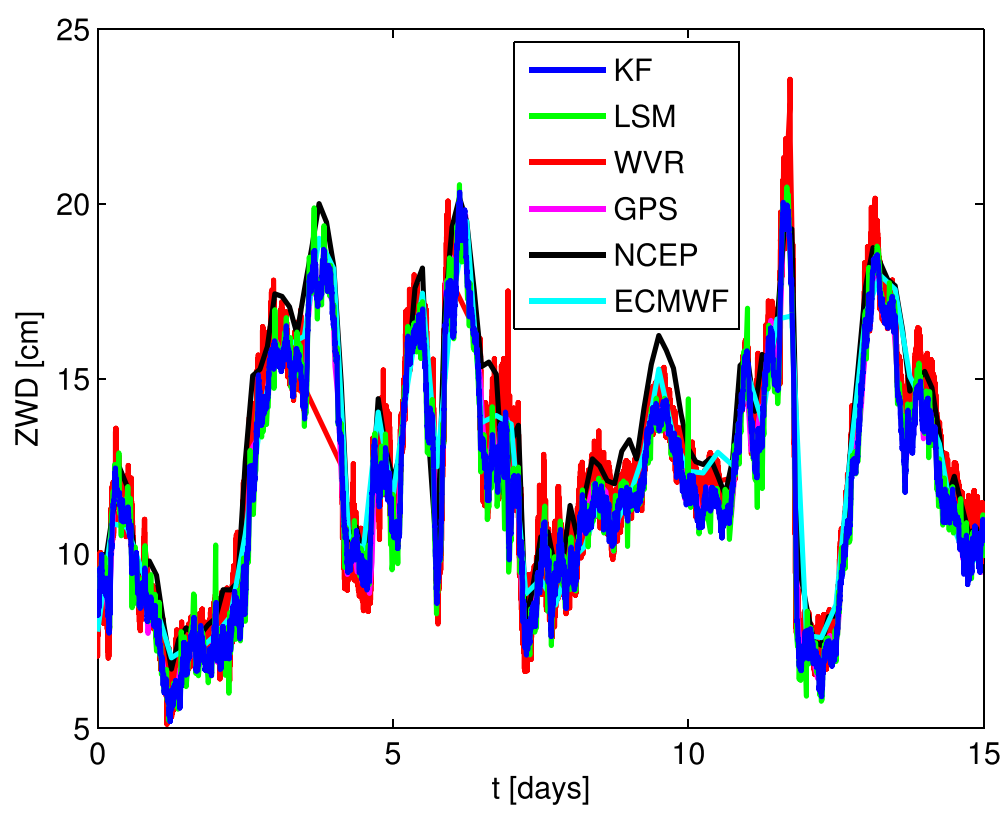

Fig. 9 ZWD comparison at Onsala during CONT11. A comparison of the ZWD from different observation techniques for station Onsala, Sweden, during the 15 days of CONT11. More details about each data set can be found in the "Data" section

same pressure data to convert ZTD to ZWD) and therefore directly compare GPS ZTD formal errors with ZWD formal errors of other techniques. For the RT solutions, no measures of uncertainty were derived.

Table 9 shows the mean ZWD formal errors for Onsala and Tsukuba during CONT11 and CONT14. While there are slight differences between the stations and campaigns, on average the $1 \mathrm{~mm}$ formal errors of WVR ZWD are significantly smaller than those of the other techniques. Tropospheric parameters from VLBI and GPS have similar formal errors around $3 \mathrm{~mm}$ with GPS having the smallest stated errors, followed by the LSM and the KF

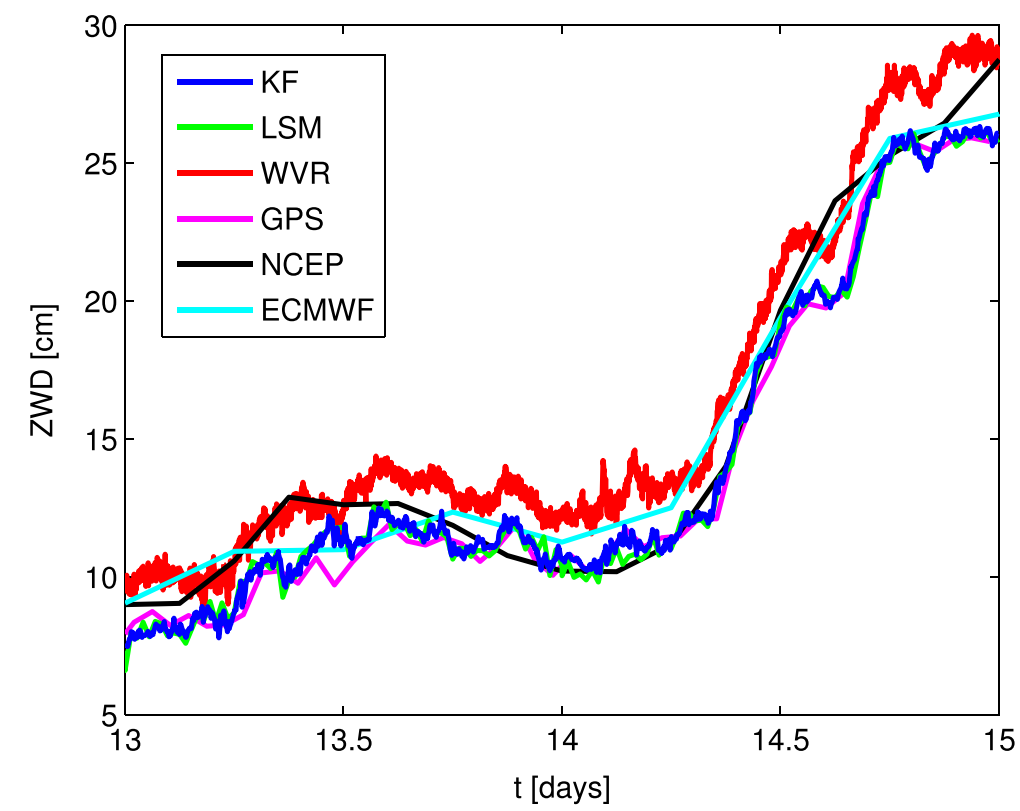

Fig. 10 ZWD comparison at Tsukuba during CONT11. The ZWD of the same techniques as in Fig. 9 are compared for station Tsukuba, Japan, during CONT11. Here, only the last 2 days are shown for better clarity 
Table 8 ZWD differences between different observation techniques. The KF and LSM solutions are both based on VLBI data, and NCEP and ECMWF are the providers of the NWM used for ray tracing. All differences are with respect to WVR data. Given are biases, RMS (without subtracting a bias), and standard deviations (bias subtracted). The values concerning the KF are valid for both station-based and constant noise approaches

\begin{tabular}{lccc}
\hline Onsala, 2011 & Bias $(\mathrm{cm})$ & RMS $(\mathrm{cm})$ & STD $(\mathrm{cm})$ \\
\hline KF-WVR & -0.47 & 0.72 & 0.55 \\
LSM-WVR & -0.46 & 0.80 & 0.65 \\
GPS-WVR & -0.43 & 0.69 & 0.54 \\
NCEP-WVR & 0.71 & 1.15 & 0.90 \\
ECMWF-WVR & 0.16 & 1.07 & 1.06 \\
\hline Tsukuba, 2011 & Bias (cm) & RMS (cm) & STD (cm) \\
\hline KF-WVR & -2.15 & 2.27 & 0.71 \\
LSM-WVR & -2.22 & 2.37 & 0.83 \\
GPS-WVR & -2.30 & 2.40 & 0.68 \\
NCEP-WVR & -1.34 & 1.98 & 1.46 \\
ECMWF-WVR & -1.59 & 2.17 & 1.48 \\
\hline Onsala, 2014 & Bias (cm) & RMS (cm) & STD (cm) \\
\hline KF-WVR & -0.71 & 0.96 & 0.65 \\
LSM-WVR & -0.71 & 0.99 & 0.69 \\
GPS-WVR & -0.89 & 1.11 & 0.66 \\
NCEP-WVR & 0.56 & 1.03 & 0.87 \\
ECMWF-WVR & -0.25 & 0.96 & 0.93 \\
\hline
\end{tabular}

VLBI solutions. Comparing the formal errors to the standard deviations obtained from ZWD differences of more than $5 \mathrm{~mm}$ in any case (Table 8), the WVR formal errors seem to be overly optimistic. The formal errors obtained in the KF solution are on average the largest and therefore probably most realistic.

\section{Conclusions}

In this study, we focused on ZWD determination by Kalman filtering VLBI data. First, we derived stochastic models that take into account station- and timedependent differences. A good agreement of the models created from time series of KF and LSM solutions as well as with those from external data of selected stations was found. The ZWD noise resembled that of a random walk more closely than that of turbulence theory, confirming previous empirical results (Jarlemark and Elgered 1998). In recent years, the ZWD noise has decreased with the most likely explanation that the quality of VLBI data has improved.

When applying our station-dependent models in the Kalman filter, the quality of station coordinates, which were also estimated, was clearly improved. Compared to a constant model, the improvement in baseline length and station coordinate repeatabilities was on average between 2 and $3 \%$ for all observational data considered.

Finally, comparing the VLBI ZWD of selected stations with those from other techniques, in particular WVR,

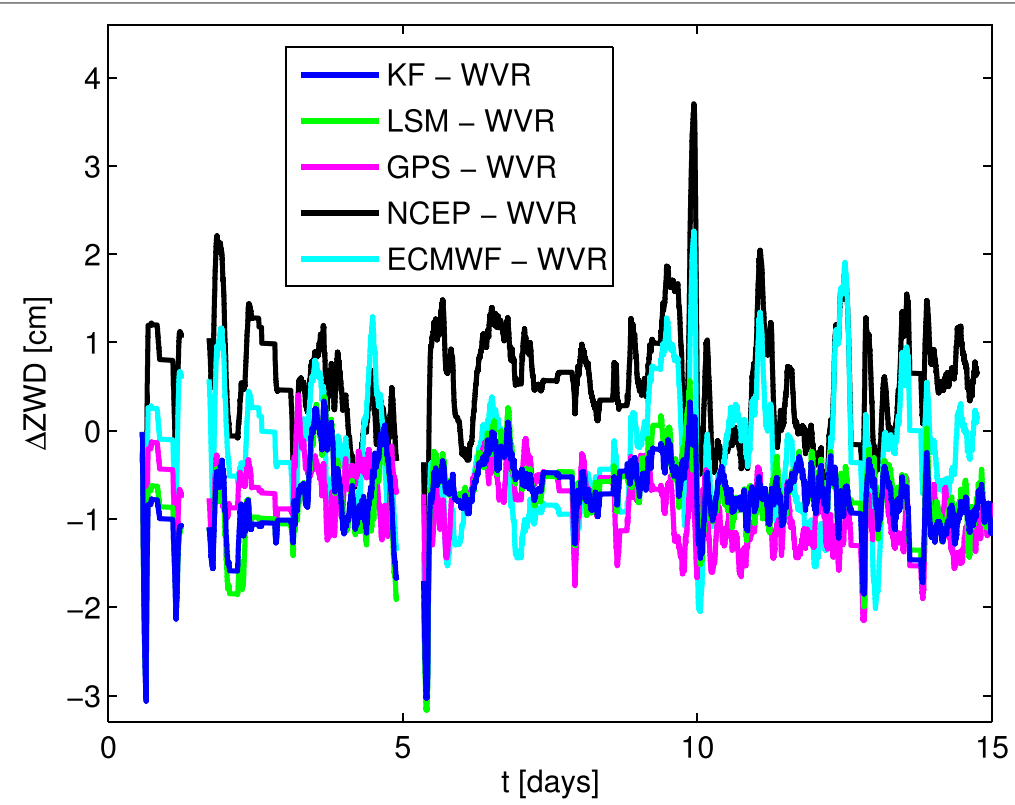

Fig. 11 ZWD differences at Onsala during CONT14. The differences in ZWD from the various techniques with respect to the WVR data are shown for station Onsala during CONT14. In order to compute the differences, all time series have been interpolated to feature the same temporal resolution as the WVR data. For a better visibility, the differences have been smoothed (see text) 
Table 9 ZWD formal errors of different observation techniques. Given are the mean formal errors in terms of 1- $\sigma$ standard deviations as obtained by the different observation techniques in units of millimeters. Both KF and LSM solutions are based on VLBI data. For GPS, the formal errors of the ZTD are given instead. No information on uncertainties is available for the ray tracing data sets

\begin{tabular}{lcccc}
\hline Data set & KF & LSM & WVR & GPS \\
\hline Onsala, CONT11 & 3.5 & 3.0 & 0.7 & 2.9 \\
Tsukuba, CONT11 & 3.7 & 2.5 & 1.4 & 3.3 \\
Onsala, CONT14 & 2.2 & 2.8 & 0.8 & 1.5 \\
\hline
\end{tabular}

showed that both KF and LSM solution have very similar biases. After removing the biases, the KF time series was unambiguously a better match to the WVR data with standard deviations smaller by $6-15 \%$.

The excellent performance of the KF allows for a promising outlook to upcoming generations of VLBI operations with VGOS, which will make real-time capability, as for instance provided by a KF, a necessity. Other VLBI analysis software developers are therefore encouraged to implement a KF as well.

In the future, it would be desirable to extend the present study by analyzing other types of VLBI sessions over a longer time span, allowing for a more comprehensive assessment of the ZWD noise characterization and the performance of a KF for ZWD determination. A detailed comparison of the VLBI results with both KF and least squares solutions of GNSS data from a larger number of collocated stations could be revealing as well. Additionally, the KF could be designed to incorporate the ZWD from other data sources to produce a combined solution, probably of higher quality than the individual contributions. Challenging would certainly be the handling of the biases between the different techniques, which were shown to be significant within the scope of this study.

\footnotetext{
Abbreviations

ASD, Allan standard deviation; ECMWF, European Centre for Medium-Range Weather Forecasts; EPOS, Earth Parameter and Orbit determination System; GFS, Global Forecast System; GFZ, German Research Centre for Geosciences; GNSS, Global Navigation Satellite Systems; GPS, Global Positioning System; GPT2, Global Pressure and Temperature 2; IERS, International Earth Rotation and Reference Systems Service; IFS, Integrated Forecast System; IGS, International GNSS Service; IVS, International VLBI Service for Geodesy and Astrometry; KF, Kalman filter; LSM, least squares method; NCEP, National Centers for Environmental Prediction; NWM, numerical weather model; PPP, precise point positioning; PSD, power spectral density; RMS, root-mean-square; RT, ray tracing; STD, standard deviation; TIGA, Tide Gauge Benchmark Monitoring; TRF, terrestrial reference frame; VGOS, VLBI Global Observing System; VieVS, Vienna VLBI Software; VLBI, very long baseline interferometry; VMF1, Vienna Mapping Functions 1; WRMS, weighted root-mean-square; WVR, water vapor radiometer; ZHD, zenith hydrostatic delay; ZTD, zenith total delay; ZWD, zenith wet delay
}

\section{Competing interests}

The authors declare that they have no competing interests.

\section{Authors' contributions}

BS implemented the Kalman filter discussed in this work, carried out the data analysis, and wrote most of the manuscript. TN contributed to the WVR processing and the results related to effects on station coordinates. MK contributed to the implementation of the Kalman filter. FZ calculated the ray-traced delays from numerical weather models. GD and ZD calculated GPS solutions for comparison. JW, RH, and HS participated in the design of the study and helped to improve the manuscript. All authors read and approved the final manuscript.

\section{Acknowledgements}

The authors would like to thank the IVS and IGS for providing the VLBI and GPS data used in this work. The access to the weather models was kindly provided through the German Weather Service "Deutscher Wetterdienst" (DWD).

Benedikt Soja and Maria Karbon work under project VLBI-ART (P 24187-N21), funded by the Austrian Science Fund (FWF).

Received: 23 April 2015 Accepted: 14 July 2015

Published online: 04 September 2015

\section{References}

Allan DW (1966) Statistics of atomic frequency standards. Proc IEEE 54(2):221-230

Altamimi Z, Collilieux X, Métivier L (2011) ITRF2008: an improved solution of the international terrestrial reference frame. J Geodesy 85(8):457-473. doi:10.1007/s00190-011-0444-4

Böhm J, Böhm S, Nilsson T, Pany A, Plank L, Spicakova H, Teke K, Schuh H (2012) The new Vienna VLBI Software VieVS. In: Kenyon S, Pacino MC, Marti U (eds). Proceedings of IAG Scientific Assembly 2009. International Association of Geodesy Symposia. Springer, Berlin Heidelberg Vol. 136. pp 1007-1011. doi:10.1007/978-3-642-20338-1_126

Böhm J, Urquhart L, Steigenberger P, Heinkelmann R, Nafisi V, Schuh H (2013) A priori gradients in the analysis of space geodetic observations. In: Altamimi Z, Collilieux X (eds). Reference Frames for Applications in Geosciences. International Association of Geodesy Symposia. Springer, Berlin Heidelberg Vol. 138. pp 105-109. doi:10.1007/978-3-642-32998-2_17.

Böhm J, Schuh H (2004) Vienna mapping functions in VLBI analyses. Geophys Res Lett 31. doi:10.1029/2003GL018984

Davis JL, Herring TA, Shapiro II, Rogers AEE, Elgered G (1985) Geodesy by radio interferometry: effects of atmospheric modeling errors on estimates of baseline length. Radio Sci 20(6):1593-1607. doi:10.1029/RS020i006p01593

Deng Z, Gendt G, Schöne T (2015) Status of the tide gauge data reprocessing at GFZ. In: Willis P (ed). Proceedings of IAG Scientific Assembly 2013. International Association of Geodesy Symposia. Springer, Berlin Heidelberg Vol. 136. in press

Dick G, Gendt G, Reigber C (2000) Operational water vapor estimation in a dense German network. In: Gowey K, Neilan R, Moore A (eds). Proceedings IGS Analysis Center Workshop. IGS 1999 Technical Reports. Jet Propul. Lab., Pasadena, Calif. pp 375-384

Dick, G, Gendt G, Reigber C (2001) First experience with near real-time water vapor estimation in a German GPS network. J Atmospheric Solar-Terrestrial Phys 63(12):1295-1304. doi:10.1016/S1364-6826(00)00248-0

Elgered, G (1993) Tropospheric radio-path delay from ground-based microwave radiometry. In: Janssen M (ed). Atmospheric Remote Sensing by Microwave Radiometry. John Wiley, New York. pp 215-258

Elgered, G, Jarlemark POJ (1998) Ground-based microwave radiometry and long-term observations of atmospheric water vapor. Radio Sci 33(3):707-717. doi:10.1029/98RS00488

Emardson TR, Jarlemark POJ (1999) Atmospheric modelling in GPS analysis and its effect on the estimated geodetic parameters. J Geodesy 73(6):322-331. doi:10.1007/s001900050249

Fey AL, Gordon D, Jacobs CS (eds) (2009) The second realization of the International Celestial Reference Frame by very long baseline interferometry. IERS Technical Note 35, Frankfurt am Main: Verlag des Bundesamtes für Kartographie und Geodäsie

Gelb A (1974) Applied optimal estimation. The MIT Press, Cambridge

Gendt G, Dick G, Söhne W (1999) GFZ analysis center of IGS - Annual report 1998. In: Gowey K, Neilan R, Moore A (eds). Analysis Center Reports. IGS 1998 Technical Reports. Jet Propul. Lab., Pasadena, Calif. pp 79-87 
Gendt G, Dick G, Reigber C, Tomassini M, Liu Y, Ramatschi M (2004) Near real time GPS water vapor monitoring for numerical weather prediction in Germany. J Meteorol Soc Jpn 82(1B):361-370

Gross RS (2000) Combinations of Earth-orientation measurements: SPACE97, COMB97, and POLE97. J Geodesy 73(12):627-637. doi:10.1007/s001900050001

Gross RS, Eubanks TM, Steppe JA, Freedman AP, Dickey JO, Runge TF (1998) A Kalman-filter-based approach to combining independent Earth-orientation series. J Geodesy 72(4):215-235. doi:10.1007/s001900050162

Hase H, Behrend D, Ma C, Petrachenko B, Schuh H, Whitney A (2012) The emerging VGOS network of the IVS. In: Behrend D, Baver KD (eds). IVS 2012 General Meeting Proceedings, NASA/CP-2012-217504. pp 8-12. http:// ivscc.gsfc.nasa.gov/publications/gm2012/hase.pdf

Heinkelmann R, Böhm J, Schuh H, Bolotin S, Engelhardt G, MacMillan DS, Negusini M, Skurikhina E, Tesmer V, Titov O (2007) Combination of long time-series of troposphere zenith delays observed by VLBI. J Geodesy 81(6-8):483-501. doi:10.1007/s00190-007-0147-z

Heinkelmann R, Böhm J, Bolotin S, Engelhardt G, Haas R, Lanotte R, MacMillan DS, Negusini M, Skurikhina E, Titov O, Schuh H (2011) VLBI-derived troposphere parameters during CONT08. J Geodesy 85(7):377-393. doi:10.1007/s00190-011-0459-x

Herring TA, Davis JL, Shapiro II (1990) Geodesy by radio interferometry: the application of Kalman Filtering to the analysis of very long baseline interferometry data. J Geophys Res Solid Earth 95(B8):12561-12581. doi:10.1029/JB095iB08p12561

Hopfield HS (1969) Two-quartic tropospheric refractivity profile for correcting satellite data. J Geophys Res 74(18):4487-4499. doi:10.1029/JC074i018p04487

Petit G, Luzum B (eds) (2010) IERS Conventions. IERS Technical Note 36, Frankfurt am Main: Verlag des Bundesamtes für Kartographie und Geodäsie

Jarlemark POJ (1997) Analysis of temporal and spatial variations in atmospheric water vapor using microwave radiometry. $\mathrm{PhD}$ thesis, Chalmers University Of Technology

Jarlemark POJ, Emardson TR, Johansson JM (1998) Wet delay variability calculated from radiometric measurements and its role in space geodetic parameter estimation. Radio Sci 33(3):719-730. doi:10.1029/98RS00551

Jarlemark POJ, Elgered G (1998) Characterizations of temporal variations in atmospheric water vapor. Geosci Remote Sensing, IEEE Trans 36(1):319-321. doi:10.1109/36.655343

Kalman RE (1960) A new approach to linear filtering and prediction problems. J Fluids Eng 82(1):11. doi:10.1115/1.3662552

Kurtenbach E, Mayer-Gürr T, Eicker A (2009) Deriving daily snapshots of the Earth's gravity field from GRACE L1B data using Kalman filtering. Geophys Res Lett 36(17):5. doi:10.1029/2009GL039564

Lagler K, Schindelegger M, Böhm J, Krásná H, Nilsson T (2013) GPT2: empirical slant delay model for radio space geodetic techniques. Geophys Res Lett 40(6):1069-1073. doi:10.1002/grl.50288

Nilsson T, Haas R (2010) Impact of atmospheric turbulence on geodetic very long baseline interferometry. J Geophys Res 115(B3407). doi:10.1029/2009JB006579

Nilsson T, Böhm J, Wijaya DD, Tresch A, Nafisi V, Schuh H (2013) Path delays in the neutral atmosphere. In: Böhm J, Schuh H (eds). Atmospheric Effects in Space Geodesy. Springer Atmospheric Sciences. Springer, Berlin Heidelberg. pp 73-136. doi:10.1007/978-3-642-36932-2_3

Nilsson T, Heinkelmann R, Karbon M, Raposo-Pulido V, Soja B, Schuh H (2014) Earth orientation parameters estimated from VLBI during the CONT11 campaign. J Geodesy 88(5):491-502. doi:10.1007/s00190-014-0700-5

Nilsson T, Soja B, Karbon M, Heinkelmann R, Schuh H (2015) Application of Kalman filtering in VLBI data analysis. Earth, Planets and Space, accepted

Pany A, Wresnik J, Böhm J, Schuh H (2007) Optimum modeling of troposphere and clock parameters in VLBI. In: Böhm J, Pany A, Schuh H (eds). Proceedings of the 18th European VLBI for Geodesy and Astrometry Working Meeting. pp 135-140. ISSN 1811-8380

Pany A, Böhm J, MacMillan D, Schuh H, Nilsson T, Wresnik J (2011) Monte Carlo simulations of the impact of troposphere, clock and measurement errors on the repeatability of VLBI positions. J Geodesy 85(1):39-50. doi:10.1007/s00190-010-0415-1

Petrachenko B, Niell A, Behrend D, Corey B, Boehm J, Charlot P, Collioud A, Gipson J, Haas R, Hobiger T, Koyama Y, MacMillan D, Malkin Z, Nilsson T, Pany A, Tuccari G, Whitney A, Wresnik J (2009) Design aspects of the
VLBI2010 system. Technical report. http://ivscc.gsfc.nasa.gov/pub/misc/ V2C/TM-2009-214180.pdf

Saastamoinen J (1972) Atmospheric correction for the troposphere and stratosphere in radio ranging of satellites. In: Henriksen SW, Mancini A, Chovitz BH (eds). American Geophysical Union, Washington, D.C. Vol. 15. pp 247-251. doi:10.1029/GM015p0247

Schöne T, Schön N, Thaller D (2009) IGS tide gauge benchmark monitoring pilot project (TIGA): scientific benefits. J Geodesy 83(3-4):249-261. doi:10.1007/s00190-008-0269-y

Schuh H, Behrend D (2012) VLBI: A fascinating technique for geodesy and astrometry. J Geodyn 61:68-80. doi:10.1016/j.jog.2012.07.007

Schuh H, Böhm J (2013) Very long baseline interferometry for geodesy and astrometry. In: Xu G (ed). Sciences of Geodesy II: Innovations and Future Developments. Springer, Berlin Heidelberg

Schüler T (2001) On ground-based GPS tropospheric delay estimation. PhD thesis, Studiengang Geodäsie und Geoinformation, Universität der Bundeswehr München

Stoew B, Rieck C (1999) Dual channel water vapour radiometer development. In: Schlüter W, Hase H (eds). Proceedings of the 13th Working Meeting on European VLBI for Geodesy and Astrometry. Verlag des Bundesamtes für Kartographie und Geodäsie, Frankfurt am Main. pp 261-264

Tralli DM, Lichten SM, Herring TA (1992) Comparison of Kalman filter estimates of zenith atmospheric path delays using the global positioning system and very long baseline interferometry. Radio Sci 27:999-1007

Treuhaft RN, Lanyi GE (1987) The effect of the dynamic wet troposphere on radio interferometric measurements. Radio Sci 22(2):251-265. doi:10.1029/RS022i002p00251

Webb FH, Zumberge JF (1993) An introduction to GIPSY/OASIS-II

Wu X, Abbondanza C, Altamimi Z, Chin TM, Collilieux X, Gross RS, Heflin MB, Jiang Y, Parker JW (2014) KALREF - A Kalman filter and time series approach to the International Terrestrial Reference Frame realization. J Geophys Res, submitted 120(5):3775-3802

Zus F, Bender M, Deng Z, Dick G, Heise S, Shang-Guan M, Wickert J (2012) A methodology to compute GPS slant total delays in a numerical weather model. Radio Sci 47(2). doi:10.1029/2011RS004853

Zus F, Dick G, Douša J, Heise S, Wickert J (2014) The rapid and precise computation of GPS slant total delays and mapping factors utilizing a numerical weather model. Radio Sci 49(3):207-216. doi:10.1002/2013RS005280

\section{Submit your manuscript to a SpringerOpen ${ }^{\circ}$ journal and benefit from:}

- Convenient online submission

Rigorous peer review

- Immediate publication on acceptance

- Open access: articles freely available online

- High visibility within the field

- Retaining the copyright to your article

Submit your next manuscript at $\boldsymbol{s p r i n g e r o p e n . c o m ~}$ 